\title{
Detecting nonclassicality and non-Gaussianity by the Wigner function and quantum teleportation in photon-added-and- subtracted two modes pair coherent state
}

\author{
Duc Minh Truong · Chuong Sy Ho · Dat Quang \\ Tran
}

Received: date / Accepted: date

\begin{abstract}
We introduce a new state called photon-added-and-subtracted two modes pair coherent state (PAASTMPCS) by simultaneously adding and subtracting photons to the different modes of a pair coherent state. Its nonclassical and non-Gaussian properties are strengthened via the negative values of its Wigner function as the numbers of adding and subtracting photons are increased. It indicates that the PAASTMPCS is an entangled state. When increasing the numbers of photon-added and photon-subtracted to a pair coherent state, the degree of entanglement in the PAASTMPCS is enhanced compared with the original pair coherent state. By using a PAASTMPCS as a non-Gaussian entangled resource, the quantum teleportation processes are studied in detail. It is shown that the number sum and phase difference measurements protocol is more appropriate than the orthogonal quadrature components measurements protocol in the quantum teleportation process of a coherent state.
\end{abstract}

Keywords Photon-added and photon-subtracted states; Pair coherent state; Wigner function; Entanglement; Quantum teleportation

PACS 42.50.Dv, 03.67.Mn, 03.67.Hk

\section{Introduction}

The addition and subtraction of photons to the classical states such as a coherent state [1] or a thermal state [2] are the normal operations that can transform to a nonclassical one [3-5]. Moreover,

Duc Minh Truong

Center for Theoretical and Computational Physics, University of Education, Hue University, Vietnam

Tel.: +84-234-3823176

Fax: $+84-234-3825824$

E-mail: tminhduc@hueuni.edu.vn

Chuong Sy Ho

${ }^{1}$ Center for Theoretical and Computational Physics, University of Education, Hue University, Vietnam

${ }^{2}$ Dong Nai University, 4 Le Quy Don, Tan Hiep, Bien Hoa City, Dong Nai, Vietnam

Dat Quang Tran

University of Transport and Communications, No.3 Cau Giay Street, Lang Thuong Ward, Dong Da District, Ha Noi, Vietnam 
these effects to the nonclassical states such as pair coherent state [6,7] or two-mode squeezing state [8] may increase the nonclassicality of the proposed states [9-12]. The schemes of generating the classes of photon-added and photon-subtracted states were proposed [13-15] and the experimental setups to generate these states in the laboratory were performed [16-18]. In continuous variables, the photon-added and photon-subtracted actions to the multi-mode Gaussian and non-Gaussian states can enhance their nonclassicality, especially their entanglement property [19-23]. Besides, the non-Gaussian entangled states can provide the potential applications in quantum information as non-Gaussian entangled resources to perform the quantum tasks such as quantum teleportation [24-26], quantum steering [27], and quantum key distribution [28, 29].

In the two modes of continuous variables systems, the adding and subtracting photons to the Gaussian states can cause these states to become non-Gaussian states. For example, the Gaussian state that is most interested in is the two-mode squeezing state [8]. The photons added and subtracted to a two-mode squeezing state have introduced many non-Gaussian states [30-33], in which the nonclassical and the entanglement properties have been investigated. These non-Gaussian states can be considered to perform many quantum tasks such as improvement of the quantum optical interferometry via the photon-added two-mode squeezed vacuum states [31], enhancement of the quantum entanglement and quantum teleportation by the multiple-photon subtraction and addition to a two-mode squeezing state $[26,32]$.

Along with continuous variables systems, the pair coherent state is a non-Gaussian state, in which its nonclassical properties were studied [7,34,35]. Moreover, the schemes for generating this state were proposed [6,36-38]. The pair coherent state is an entangled state, and the quantitative measures of entanglement, quantum teleportation, and quantum key distribution by using this state were also studied [39-43]. The photon addition to two modes of pair coherent state as photon-added pair coherent state [44] and generalized photon-added pair coherent state [45] were introduced. Furthermore, the photon-added and photon-subtracted pair coherent state [46], in which the added or subtracted photons only take place in one mode of the pair coherent state was also introduced. In these states, the nonclassical and the entanglement properties of them were studied in detail. Because the pair coherent state is a non-Gaussian state, the proposed states by adding or subtracting photons to this state are non-Gaussian states as well, and the non-Gaussian characteristics of them can be strengthened.

In this paper, so as to extend simultaneously adding and subtracting photons to both modes of a pair coherent state, we introduce a new state called photon-added-and-subtracted two modes pair coherent state (PAASTMPCS) in Section 2. We show the nonclassical and non-Gaussian characteristics of the new state by examining the Wigner function in Section 3 and the entanglement degree by using the linear entropy of the PAASTMPCS in Section 4. We use the PAASTMPCS as a non-Gaussian resource for the quantum teleportation processes by using the orthogonal quadrature components measurements protocol in Section 5, and the number sum and phase difference measurements protocol in Section 6. Finally, the main results of the paper are summarized in the conclusions.

\section{Photon-added-and-subtracted two modes pair coherent state}

The pair coherent state (PCS) $|\xi, q\rangle_{a b}$ is a state of the two-mode radiation field [7], which is the eigenstate of both the boson annihilation operators $\hat{a} \hat{b}$ and the charge operator $\hat{Q}=\hat{b}^{\dagger} \hat{b}-\hat{a}^{\dagger} \hat{a}$ as 
follows

$$
\begin{aligned}
\hat{a} \hat{b}|\xi, q\rangle_{a b} & =\xi|\xi, q\rangle_{a b}, \\
\hat{Q}|\xi, q\rangle_{a b} & =q|\xi, q\rangle_{a b},
\end{aligned}
$$

where $\xi=|\xi| \mathrm{e}^{i \phi}$ is a complex number with $\phi$ real, $q$ is an integer referred to the difference in the number of photons between modes $a$ and $b$. In case $q \geq 0$, in the term of Fock states, $|\xi, q\rangle_{a b}$ is written as

$$
|\xi, q\rangle_{a b}=\left(\sum_{m=0}^{\infty} \frac{|\xi|^{2 m}}{m !(m+q) !}\right)^{-1 / 2} \sum_{n=0}^{\infty} \frac{\xi^{n}}{[n !(n+q) !]^{1 / 2}}|n, n+q\rangle_{a b}
$$

$|n, n+q\rangle_{a b}$ is a two-mode Fock state.

Now, we introduce a new state called photon-added-and-subtracted two modes pair coherent state (PAASTMPCS) by acting $k$ times of creation operator $\hat{a}^{\dagger}$ on mode $a$ and $l$ times with $l \leq q$ of annihilation operator $\hat{b}$ on mode $b$ of a PCS $|\xi, q\rangle_{a b}$ as

$$
|\xi, q ; k, l\rangle_{a b}=B_{q ; k, l}(\xi) \hat{a}^{\dagger k} \hat{b}^{l}|\xi, q\rangle_{a b}
$$

where $k$ and $l$ are non-negative integers and the normalized factor $B_{q ; k, l}(\xi)$ is determined by

$$
B_{q ; k, l}(\xi)^{-2}=\sum_{m=0}^{\infty} \frac{|\xi|^{2 m}(m+k) !}{(m !)^{2}(m+q-l) !}
$$

It should be noted that, the PAASTMPCS in the form of $|\xi, q ; k, l\rangle_{a b}$ in Eqs. (4) and (5) does not explicitly depend on $q$ or on $l$ but it only depends on the difference between $q$ and $l$. Therefore, we set $h=q-l$, the PAASTMPCS is written as $|\xi, k, h\rangle_{a b}$ in the term of two-mode Fock states in the form

$$
|\xi, q ; k, l\rangle_{a b} \equiv|\xi, k, h\rangle_{a b}=\sum_{n=0}^{\infty} C_{n ; k, h}(\xi)|n+k, n+h\rangle_{a b}
$$

where

$$
C_{n ; k, h}(\xi)=\left(\sum_{m=0}^{\infty} \frac{|\xi|^{2 m}(m+k) !}{(m !)^{2}(m+h) !}\right)^{-1 / 2} \xi^{n} \sqrt{\frac{(n+k) !}{(n !)^{2}(n+h) !}} .
$$

When $k=0$ and $h=q$, or $k=l=0$, the PAASTMPCS is reduced to the PCS [6]. In what follows, we will use PAASTMPCS in the form of $|\xi, k, h\rangle_{a b}$ in Eqs. (6) and (7) for calculation. 


\section{Wigner function}

In quantum optics, a Wigner function is used to describe a quantum state in the phase space. In some states, their Wigner function can take the negative values. Via the negative values of its Wigner function, it confirms a certain state is a nonclassical and non-Gaussian state $[20,47,48]$. In the PAASTMPCS, the Wigner function can be given in the term of coherent states as

$$
\begin{aligned}
W= & \frac{4 e^{2\left(\left|\alpha_{a}\right|^{2}+\left|\alpha_{b}\right|^{2}\right)}}{\pi^{4}} \int d^{2} \gamma_{a} d^{2} \gamma_{b} e^{2\left(\gamma_{a}^{*} \alpha_{a}+\gamma_{b}^{*} \alpha_{b}-\gamma_{a} \alpha_{a}^{*}-\gamma_{b} \alpha_{b}^{*}\right)} \\
& \times{ }_{b a}\left\langle-\gamma_{b},-\gamma_{a}\left|\hat{\rho}_{a b}\right| \gamma_{a}, \gamma_{b}\right\rangle_{a b},
\end{aligned}
$$

where $\alpha_{a}$ and $\alpha_{b}$ are complex numbers in the phase space, $\left|\gamma_{a}\right\rangle_{a}$ and $\left|\gamma_{b}\right\rangle_{b}$ denote the coherent states, and $\hat{\rho}_{a b}$ is the density operator of the PAASTMPCS. Using Eq. (6), the density operator $\hat{\rho}_{a b}$ is written as

$$
\hat{\rho}_{a b}=|\xi, k, h\rangle_{a b}\left\langle\xi, k, h\left|=\sum_{n, m=0}^{\infty} C_{n ; k, h}(\xi) C_{m ; k, h}^{*}(\xi)\right| n+k, n+h\right\rangle_{a b}\langle m+h, m+k| .
$$

Substituting the density operator $\hat{\rho}_{a b}$ in Eq. (9) into Eq. (8) then calculating the complex integrals (see Appendix), we obtain the Wigner function of the PAASTMPCS in the form

$$
\begin{aligned}
W= & \frac{4 e^{-2\left(\left|\alpha_{a}\right|^{2}+\left|\alpha_{b}\right|^{2}\right)}}{\pi^{2}}\left[\sum_{j=0}^{\infty} \frac{|\xi|^{2 j}(j+k) !}{(j !)^{2}(j+h) !}\right]^{-1} \\
& \times \sum_{m, n=0}^{\infty} \frac{\left(4\left|\xi \alpha_{a} \alpha_{b}\right|\right)^{m+n} \cos \left[(m-n)\left(\varphi_{a}+\varphi_{b}-\phi\right)\right]\left|2 \alpha_{a}\right|^{2 k}\left|\alpha_{b}\right|^{2 h}}{m ! n !(m+h) !(n+h) !} \\
& \times{ }_{2} F_{0}\left[-n-k,-m-k ; ;-1 /\left(2\left|\alpha_{a}\right|\right)^{2}\right]_{2} F_{0}\left[-n-h,-m-h ; ;-1 /\left(2\left|\alpha_{b}\right|\right)^{2}\right],
\end{aligned}
$$

where $\alpha_{a}=\left|\alpha_{a}\right| e^{i \varphi_{a}}, \alpha_{b}=\left|\alpha_{b}\right| e^{i \varphi_{b}}$, and ${ }_{2} F_{0}$ denotes the hypergeometric function.

We use the analytical expression in Eq. (10) to investigate the nonclassical and non-Gaussian behaviours in the PAASTMPCS. In Fig. 1, we plot the dependence of the Wigner function $W$ as a function of both real and imaginary parts of $\alpha_{a}$ with $|\xi|=0.2, \alpha_{b}=0.5, \phi=0$, and $h=k=1$. The result shows that the Wigner function of the PAASTMPCS gets negative values in some regions of the phase space. Therefore, we conclude that the PAASTMPCS is a nonclassical and non-Gaussian state. Besides, in the region corresponding to the appropriate values of $|\xi|$, the depth of the Wigner function can become higher when increasing the numbers of photon-added $k$ and photon-subtracted $l$ (see Fig. 2). The blue solid line $(k=0, h=q=6)$ corresponding to the PCS is the least negative. The other lines corresponding to the PAASTMPCS are more negative compared with the PCS. This proves that adding and subtracting photons to the different modes are very important in enhancing the nonclassical and non-Gaussian properties of the PAASTMPCS compared with the original PCS.

\section{Linear entropy}

In two modes states, some criteria can be used effectively to detect the entanglement [49-51] and the degree of entanglement $[39,52,53]$ of them. In order to investigate the degree of entanglement in 


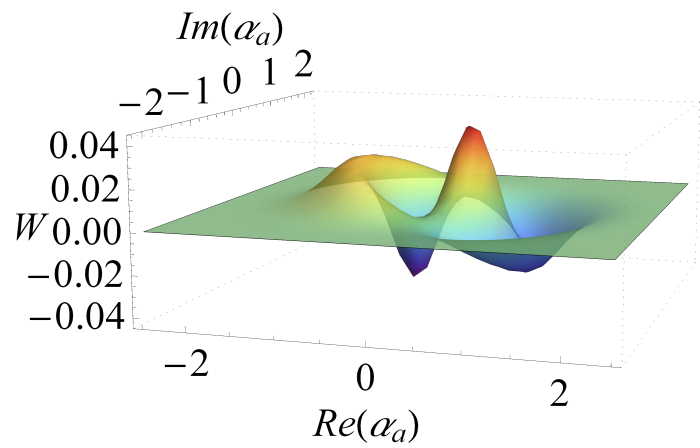

Fig. 1 The Wigner function $W$ as a function of real and imaginary parts of $\alpha_{a}$ with $|\xi|=0.2, \alpha_{b}=0.5, \phi=0$, and $h=k=1$.

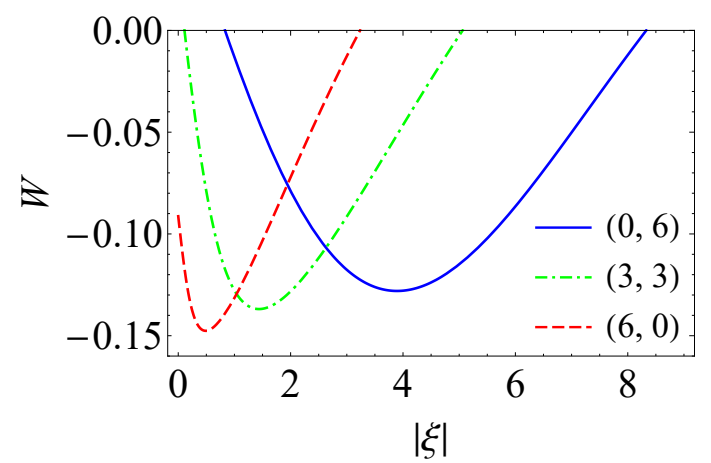

Fig. 2 The Wigner function $W$ as a function of $|\xi|$ with $\left|\alpha_{a}\right|=0.21,\left|\alpha_{b}\right|=0.26, \varphi_{a}+\varphi_{b}-\phi=\pi$ for $(k, h)=(0,6)$ (the blue solid line), (3,3) (the green dot-dashed curve), and $(6,0)$ (the red dashed curve).

the PAASTMPCS, we use the linear entropy criterion [39]. Accordingly, the entanglement degree coefficient $E_{\text {lin }}$ is given in the form

$$
E_{\text {lin }}=1-\operatorname{Tr}\left(\widehat{\rho}_{k}^{2}\right)
$$

where $\operatorname{Tr}$ is denoted as the trace of the matrix. A state is entangled if $E_{\text {lin }}>0$. When $E_{\text {lin }}=1$, the state becomes maximum entangled. For the PAASTMPCS, from the density operator $\hat{\rho}_{a b}$ was given in Eq. (9), we get

$$
\begin{aligned}
& \hat{\rho}_{a}^{2}=\sum_{n=0}^{\infty}\left|C_{n ; k, h}(\xi)\right|^{4}|n+k\rangle_{a}\langle n+k|, \\
& \hat{\rho}_{b}^{2}=\sum_{n=0}^{\infty}\left|C_{n ; k, h}(\xi)\right|^{4}|n+h\rangle_{b}\langle n+h| .
\end{aligned}
$$


From Eqs. (12) and (13), we have $\operatorname{Tr}\left(\widehat{\rho}_{a}^{2}\right)=\operatorname{Tr}\left(\widehat{\rho}_{b}^{2}\right)$. It is easy to obtain the linear entropy $E_{\text {lin }}$ of the PAASTMPCS as follows

$$
\begin{aligned}
E_{\text {lin }} & =1-\sum_{n=0}^{\infty}\left|C_{n ; k, h}(\xi)\right|^{4} \\
& =1-\left(\sum_{m=0}^{\infty} \frac{|\xi|^{2 m}(m+k) !}{(m !)^{2}(m+h) !}\right)^{-2} \sum_{n=0}^{\infty}\left(\frac{|\xi|^{2 n}(n+k) !}{(n !)^{2}(n+h) !}\right)^{2} .
\end{aligned}
$$

We examine the entanglement degree of the PAASTMPCS by using Eq. (14). In Fig. 3, we plot the dependence of $E_{\operatorname{lin}}$ on $|\xi|$ for several values of $k$ and $h=q-l$, therein, the case of $k=0$ and $h=q=6$ (the solid blue line) corresponds to the PCS, and the others are the PAASTMPCS. At the same values of $|\xi|$, the curves on the graphs show that the entanglement degree coefficient $E_{\text {lin }}$ in the PAASTMPCS is always higher than that in the original PCS. Besides, the curves in Figs. 3 (a) and (b) show the entanglement degree coefficient $E_{\text {lin }}$ increases if the numbers of photon-added $k$ and photon-subtracted $l$ increase (i.e., $h$ decreases). It means that the degree of entanglement of the PAASTMPCS is enhanced by simultaneously increasing the number of photon-added and photon-subtracted to the original PCS. In addition, in the case of adding and subtracting more photons, the value of the entanglement degree coefficient $E_{\text {lin }}$ is increasing, especially in the case of the difference of $k-h$ is getting bigger and bigger (see Fig. 3 (c)).

\section{Quantum teleportation uses the orthogonal quadrature components measurements protocol}

In continuous variables quantum teleportation, both sender (Alice) and receiver (Bob) must be shared by a two-mode entangled state. In this section, we use the PAASTMPCS as an entanglement resource for teleportation by exploiting the orthogonal quadrature components measurements protocol [54]. In this first protocol, we assume that Alice possesses the mode $a$ and Bob holds the mode $b$. Alice needs to teleport to Bod a coherent state $|\alpha\rangle_{c}$ of mode $c$, in which this state is expanded in the term of the Fock states as

$$
|\alpha\rangle_{c}=\sum_{m=0}^{\infty} d_{m}|m\rangle_{c}
$$

with $d_{m}=e^{-|\alpha|^{2} / 2} \alpha^{m} / \sqrt{m !}$. The input state of the system is written as

$$
\left|\Phi_{\text {in }}\right\rangle_{a b c}=|\xi, k, h\rangle_{a b}|\alpha\rangle_{c}=\sum_{m=0}^{\infty} \sum_{n=0}^{\infty} C_{n ; k, h}(\xi) d_{m}|n+k, n+h\rangle_{a b}|m\rangle_{c}
$$

where $C_{n ; k, h}(\xi)$ is given by Eq. (7). Next, Alice measures the orthogonal quadrature components on two modes $a$ and $c$. After this measurement, the state of Bob (non-normalized) becomes

$$
|\Phi\rangle_{b}={ }_{a c}\langle\beta \mid \xi, k, h\rangle_{a b} \otimes|\alpha\rangle_{c}
$$

where $|\beta\rangle_{a c}$ is the eigenstate of two commutative operators $\hat{x}_{-}=\hat{x}_{c}-\hat{x}_{a}$ and $\hat{y}_{+}=\hat{y}_{a}+\hat{y}_{c}$ [54]. Such state is expressed in the term of the Fock states as

$$
|\beta\rangle_{a c}=\frac{1}{\sqrt{\pi}} \sum_{m=0}^{\infty} \hat{D}_{c}(\beta)|m, m\rangle_{a c},
$$



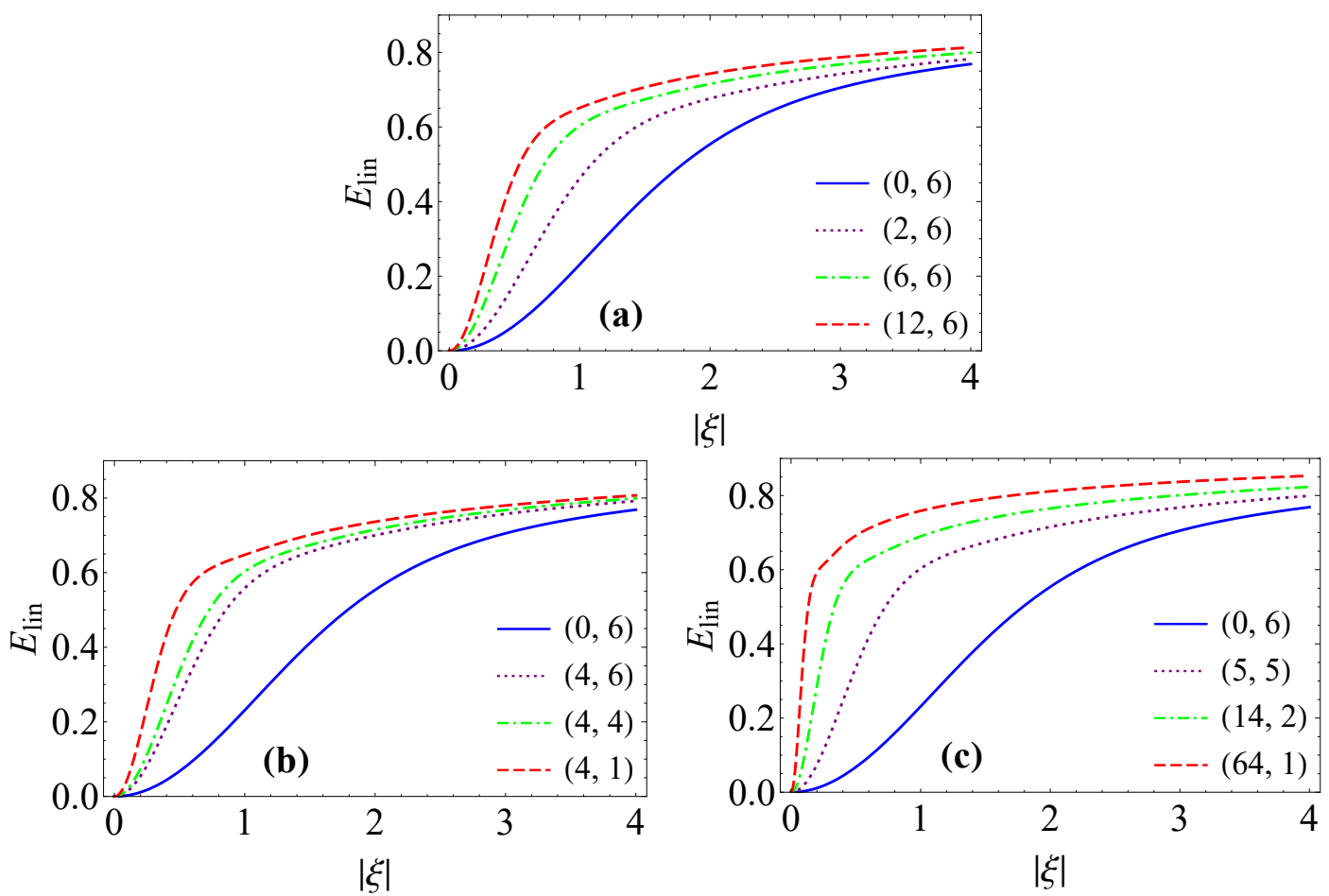

Fig. 3 The linear entropy $E_{\text {lin }}$ as a function of $|\xi|$ for in $(a)(k, h)=(0,6)$ (the blue solid line), (2, 6) (the purple dotted curve), (6, 6) (the green dot-dashed curve), and $(12,6)$ (the red dashed curve), in $(b)(k, h)=(0,6)($ the blue solid line), (4,6) (the purple dotted curve), (4, 4) (the green dot-dashed curve), and (4, 1) (the red dashed curve), and in $(c)(k, h)=(0,6)$ (the blue solid line), (5, 5) (the purple dotted curve), (14, 2) (the green dot-dashed curve), and $(64,1)$ (the red dashed curve).

with $\hat{x}_{-}|\beta\rangle_{a c}=\operatorname{Re}(\beta)|\beta\rangle_{a c}, \quad \hat{y}_{+}|\beta\rangle_{a c}=\operatorname{Im}(\beta)|\beta\rangle_{a c}$, where $\hat{D}_{c}(\beta)$ is denoted as the displacement operator acting on the state of mode $c$. The state in the Eq. (17) is expanded in normalized form as follows

$$
\left|\Phi_{\mathrm{nor}}\right\rangle_{b}=\frac{e^{-|\alpha-\beta|^{2} / 2} e^{\left(\alpha \beta^{*}-\alpha^{*} \beta\right) / 2}}{\sqrt{P(\beta) \pi}} \sum_{n=0}^{\infty} C_{n ; k, h}(\xi) \frac{(\alpha-\beta)^{n+k}}{\sqrt{(n+k) !}}|n+h\rangle_{b},
$$

where $P(\beta)$ is the probability of the measurement given by

$$
P(\beta)=\frac{e^{-|\alpha-\beta|^{2}}}{\pi} \sum_{n=0}^{\infty}\left|C_{n ; k, h}(\xi)\right|^{2} \frac{|\alpha-\beta|^{2(n+k)}}{(n+k) !} .
$$

The results of the measurement are sent to Bob by a classical channel. Finally, Bob restores the teleported state by using the operator $\hat{D}(\beta)$. The quantum teleportation process is completed. The output state (normalized) reads

$$
\left|\Psi_{\text {nor }}\right\rangle_{\text {out }}=\frac{e^{-|\alpha-\beta|^{2} / 2} e^{\left(\alpha \beta^{*}-\alpha^{*} \beta\right) / 2}}{\sqrt{P(\beta) \pi}} \sum_{n=0}^{\infty} C_{n ; k, h}(\xi) \frac{(\alpha-\beta)^{n+k}}{\sqrt{(n+k) !}} \hat{D}(\beta)|n+h\rangle .
$$


The quality of the quantum teleportation process is shown by the average fidelity. The fidelity is an overlap of the output and input states

$$
F=\left.\left.\right|_{c}\left\langle\alpha \mid \Phi_{\text {nor }}\right\rangle_{\text {out }}\right|^{2}=\frac{e^{-2|\alpha-\beta|^{2}}}{P(\beta) \pi} \sum_{n, m=0}^{\infty} \frac{C_{n ; k, h}(\xi) C_{m ; k, h}^{*}(\xi)|\alpha-\beta|^{2(n+m+k+h)}}{\sqrt{(n+k) !(n+h) !(m+k) !(m+h) !}} .
$$

Therefore, the average fidelity is determined as

$$
F_{\mathrm{av}}=\int P(\beta) F d^{2} \beta
$$

The quantum teleportation process is successful when $F_{\mathrm{av}}>0.5$ and perfect at $F_{\mathrm{av}}=1$. The our calculated result is

$$
F_{\mathrm{av}}=\sum_{m, n=0}^{\infty} \frac{C_{n ; k, h}(\xi) C_{m ; k, h}^{*}(\xi)(n+m+k+h) !}{2^{n+m+k+h+1} \sqrt{(n+k) !(n+h) !(m+k) !(m+h) !}} .
$$

In case $\xi=|\xi|$, the average fidelity in Eq. (24) is explicitly written as

$$
F_{\mathrm{av}}=\left(\sum_{j=0}^{\infty} \frac{|\xi|^{2 j}(j+k) !}{(j !)^{2}(j+h) !}\right)^{-1} \sum_{m, n=0}^{\infty} \frac{2^{-(n+m+k+h+1)}|\xi|^{n+m}(n+m+k+h) !}{n ! m !(n+h) !(m+h) !} .
$$

From Eq. (25) it is easy to see that the average fidelity $F_{\text {av }}$ does not depend on the amplitude $|\alpha|$ of a coherent state to be teleported. In Fig. 4, the curves show the dependence of the average fidelity $F_{\text {av }}$ as a function of $|\xi|$ with the different values of parameters $k$ and $h$, in which, the case $k=0$ and $h=q=6$ (the solid blue line) corresponds to the PCS, and the others are the PAASTMPCS. It is clear that the average fidelity in the PAASTMPCS is always bigger than that in the PCS. More importantly, when the parameter $|\xi|$ is increasing, the average fidelity in the PAASTMPCS is improved by increasing the number of photon added and photon subtracted to the PCS. That is shown in Figs. 4 (a) and (b). Besides, the average fidelity $F_{\text {av }}$ gets the biggest values in case the number of photons in mode $a$ and mode $b$ are equal, i.e., $h=k=q-l$ (see Fig. 4 (c)). Thus, the role of adding and subtracting photons is very important in enhancing the degree of average fidelity of the quantum teleportation process by using the first protocol.

\section{Quantum teleportation uses the number sum and phase difference measurements protocol}

In the second protocol as the number sum and phase difference measurements [55], the input state of the system is also given by Eq. (16). Now, Alice measures the photon number sum and phase difference on two modes $a$ and $c$, the state of the system becomes

$$
|\Phi\rangle_{b}=P_{a c}^{-1 / 2}\left\langle\phi_{N}^{-} \mid \Phi_{\text {in }}\right\rangle_{a b c}
$$



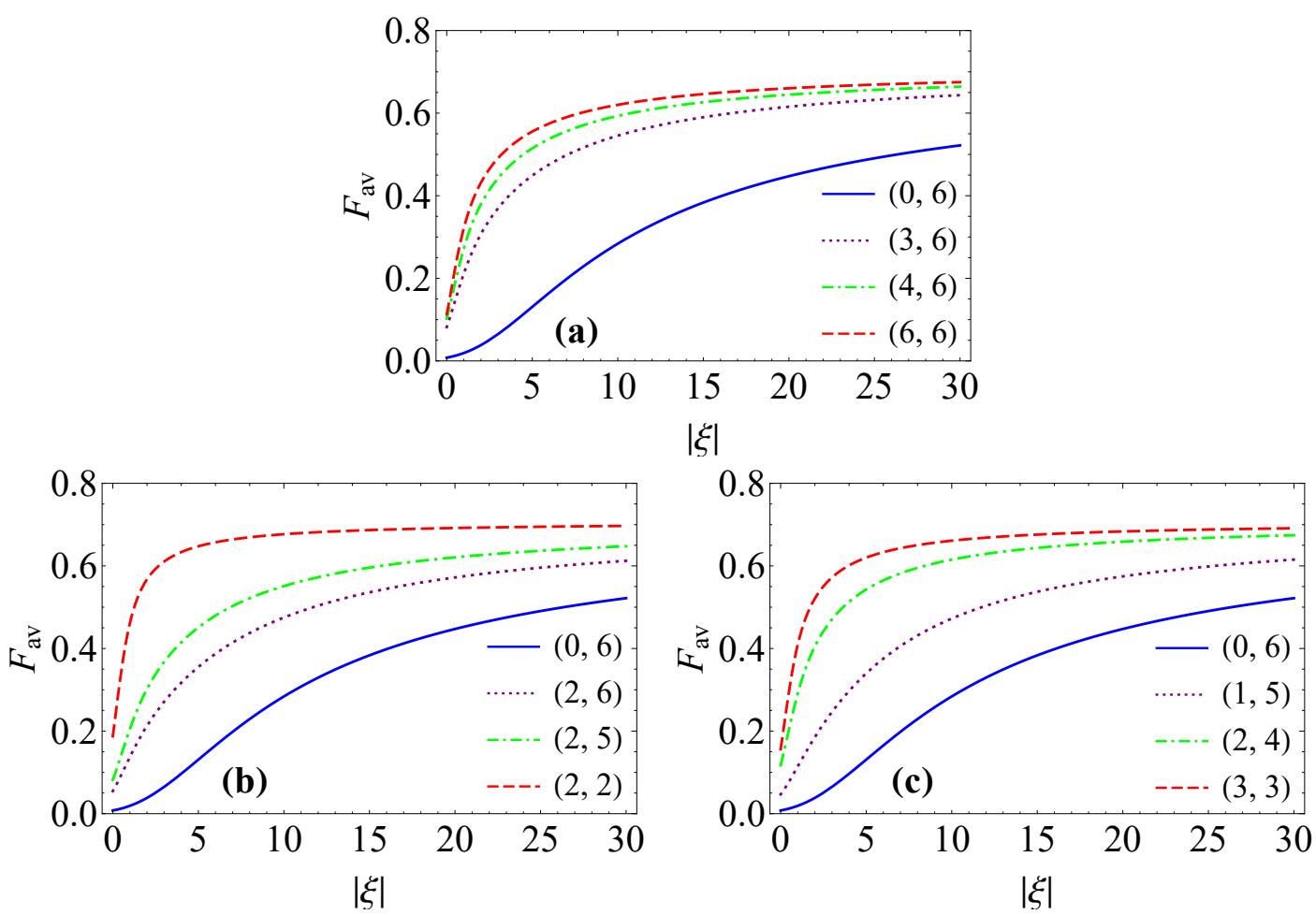

Fig. 4 The average fidelity $F_{a v}$ as a function of $|\xi|$ for in $(a)(k, h)=(0,6)$ (the blue solid line), $(3,6)$ (the purple dotted curve), $(4,6)$ (the green dot-dashed curve), and $(6,6)$ (the red dashed curve), in (b) $(k, h)=(0,6)$ (the blue solid line), (2,6) (the purple dotted curve), (2, 5) (the green dot-dashed curve), and (2, 2) (the red dashed curve), and in $(c)(k, h)=(0,6)$ (the blue solid line), $(1,5)$ (the purple dotted curve), (2, 4) (the green dot-dashed curve), and $(3,3)$ (the red dashed curve).

where $P$ is the probability to obtain the photon number sum $N$ and the phase difference $\phi^{-}$, and $\left|\phi_{N}^{-}\right\rangle_{a c}$ is the eigenstate of the photon number sum operator $\hat{N}=\hat{N}_{a}+\hat{N}_{c}$ and the phase difference operator $\hat{\phi}^{-}=\hat{\phi}_{a}-\hat{\phi}_{c}$. Such state reads [56]

$$
\left|\phi_{N}^{-}\right\rangle_{a c}=\frac{1}{\sqrt{2 \pi}} \sum_{j=0}^{N} e^{i j \phi^{-}}|j\rangle_{a}|N-j\rangle_{c}
$$

in which, $\phi^{-}$is restricted in the windows $\phi_{0}^{-} \leq \phi^{-}<\phi_{0}^{-}+2 \pi, \phi_{0}^{-}$is an arbitrary real number. We clearly write the Eq. (26) as

$$
\left|\Phi_{b}\right\rangle=(2 \pi P)^{-1 / 2} \sum_{n=0}^{N-k} e^{-i(n+k) \phi^{-}} C_{n ; k, h}(\xi) d_{N-(n+k)}|n+h\rangle_{b},
$$

with $P=\frac{1}{2 \pi} \sum_{n=0}^{N-k}\left|C_{n ; k, h}(\xi)\right|^{2}\left|d_{N-(n+k)}\right|^{2}$. After the measurement, Alice sends to Bob the numbers of $N$ and $\phi^{-}$by a classical channel. Based on these data, Bob rotates his phase by using the 
unitary operator $\hat{U}=e^{i\left(\hat{N}_{b}+k-h\right) \phi^{-}}$with $\hat{N}_{b}$ is the photon number operator of mode $b$. The state of Bob becomes

$$
|\Psi\rangle=\hat{U}\left|\Phi_{b}\right\rangle=(2 \pi P)^{-1 / 2} \sum_{n=0}^{N-k} C_{n ; k, h}(\xi) d_{N-(n+k)}|n+h\rangle_{b}
$$

Then, Bob transforms the photon number $n+h$ to become $N-(n+k)$. The teleportation process is completed. The output state is

$$
|\Psi\rangle_{\text {out }}=(2 \pi P)^{-1 / 2} \sum_{n=0}^{N-k} C_{n ; k, h}(\xi) d_{N-(n+k)}|N-(n+k)\rangle_{b} .
$$

In order to estimate the efficacy of this teleportation process, we also use the average fidelity. The fidelity of the quantum teleportation process is determined by

$$
F=\left.\left.\right|_{c}\langle\alpha \mid \Phi\rangle_{\text {out }}\right|^{2}=\left.\left.\frac{1}{2 \pi P}\left|\sum_{n=0}^{N-k} C_{n ; k, h}(\xi)\right| d_{N-(n+k)}\right|^{2}\right|^{2} .
$$

From that, the average fidelity is represented by

$$
F_{\mathrm{av}}=\sum_{N=k}^{\infty} \int_{\phi_{0}^{-}}^{\phi_{0}^{-}+2 \pi} P F d \phi^{-}=\left.\left.\sum_{N=k}^{\infty}\left|\sum_{n=0}^{N-k} C_{n ; k, h}(\xi)\right| d_{N-(n+k)}\right|^{2}\right|^{2} .
$$

Using Eq. (32) with the assumption that $\xi=|\xi|$, we obtain the result as follows

$$
F_{\mathrm{av}}=\left(\sum_{j=0}^{\infty} \frac{|\xi|^{2 j}(j+k) !}{(j !)^{2}(j+h) !}\right)^{-1} \sum_{m=0}^{\infty}\left(\sum_{n=0}^{m} \frac{|\xi|^{n}|\alpha|^{2(m-n)} \sqrt{(n+k) !}}{e^{|\alpha|^{2} n !(m-n) ! \sqrt{(n+h) !}}}\right)^{2} .
$$

It is clear in Eq. (33) that the average fidelity $F_{\text {av }}$ not only depends on the parameters in the PAASTMPCS, but also depends on the amplitude $|\alpha|$ of a teleported state. In Fig. 5, we plot the dependence of the average fidelity $F_{\text {av }}$ on $|\xi|$ with several values of $|\alpha|$ when $k=4$ and $h=6$. We see that the average fidelity $F_{\text {av }}$ increases with the decreasing of $|\alpha|$, and it can approach to unit if $|\alpha|=0.25$ and $|\xi|$ is large enough. In order to compare the degree of average fidelity $F_{\text {av }}$ between PAASTMPCS and PCS, we plot the dependence of the average fidelity $F_{\mathrm{av}}$ on $|\xi|, k$ and $h$ for $|\alpha|=1.00$ in Fig. 6 . The case $k=0, h=q=6$ (the blue solid line) corresponds to the PCS, and the others are the PAASTMPCS. When increasing the numbers of photon-added and photon-subtracted to both modes $a$ and $b$ of PCS, the average fidelity $F_{\text {av }}$ in the PAASTMPCS is always higher than that in the PCS (see Figs. 6 (a) and (b)). It proves that the average fidelity of the quantum teleportation process is enhanced by adding and subtracting photons. These indicate the important role of the photon addition and photon subtraction operation to enhance the degree of average fidelity of the quantum teleportation process by using the second protocol, where the entangled resource is the PAASTMPCS. 


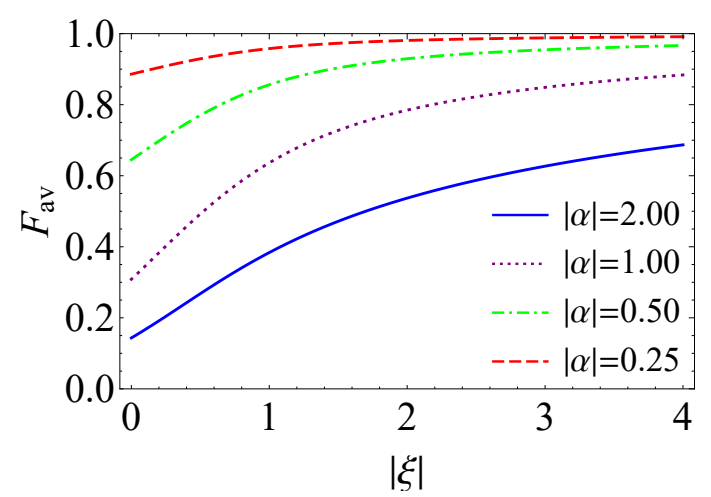

Fig. 5 The average fidelity $F_{a v}$ as a function of $|\xi|$ for $k=4, h=6$ and $|\alpha|=2.00$ (the blue solid line), $|\alpha|=1.00$ (the purple dotted curve), $|\alpha|=0.50$ (the green dot-dashed curve), and $|\alpha|=0.25$ (the red dashed curve).
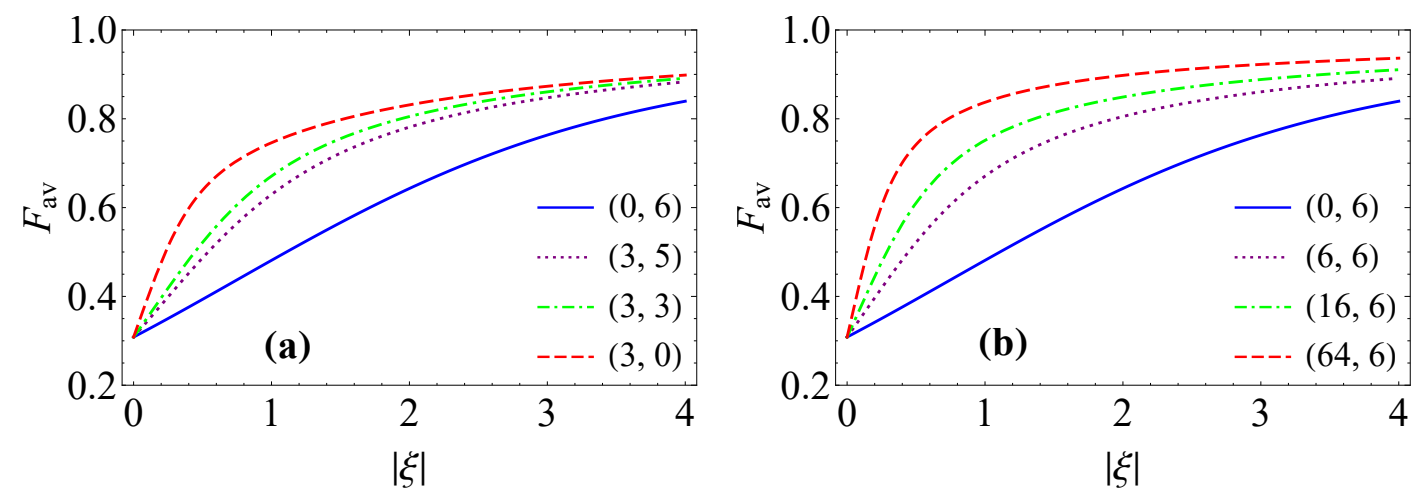

Fig. 6 The average fidelity $F_{a v}$ as a function of $|\xi|$ for $|\alpha|=1.00$ in $(a)(k, h)=(0,6)$ (the blue solid line), (3, 5) (the purple dotted curve), (3, 3) (the green dot-dashed curve), and (3, 0) (the red dashed curve), and in (b) $(k, h)=(0,6)$ (the blue solid line), $(6,6)$ (the purple dotted curve), (16,6) (the green dot-dashed curve), and (64, 6) (the red dashed curve).

\section{Conclusions}

In this paper, we have introduced a new state called photon-added-and-subtracted two modes pair coherent state (PAASTMPCS) and studied their nonclassical and non-Gaussian properties based on the Wigner function. It is shown that the Wigner function of the PAASTMPCS gets negative values in some regions of the phase space and depends on the adding and subtracting photons to the PCS. It proves that the PAASTMPCS is a nonclassical and non-Gaussian state. The nonclassical and non-Gaussian properties of this state are enhanced by adding and subtracting photons to the original PCS. We have quantified the entanglement degree of PAASTMPCS based on the linear entropy criterion. It is shown that the PAASTMPCS is an entangled state, and the entanglement degree of this state is greater than that of the original PCS. The more photon-added and photon-subtracted are, the more increasing the entanglement degree is. The entanglement degree can approach to the unit when the amplitude of the PAASTMPCS $|\xi|$ as well as the numbers of adding and subtracting photons are very large. It proves that the PAASTMPCS is 
a non-Gaussian resource for performing quantum teleportation process. For the first protocol by using the orthogonal quadrature components measurements, the average fidelity of the quantum teleportation process is always bigger than that of the original PCS. Moreover, the average fidelity gets the maximum values in case the numbers of photons in mode $a$ and mode $b$ are equal, i.e., the photon-added $k$ and photon-subtracted $l$ satisfy the condition $k+l=q$. For the second protocol by using the number sum and phase difference measurements, it is shown that the average fidelity can approach to the unit when the amplitude $|\alpha|$ of the input state $|\alpha\rangle_{c}$ is small and the amplitude $|\xi|$ of the PAASTMPCS is not large. For example, when $|\alpha|=0.25$, and $|\xi|=4$, the $F_{\text {av }}$ reaches 0.998. Besides, if the numbers of photon-added and photon-subtracted to the PCS increase, the average fidelity $F_{\text {av }}$ also increases and the value of $F_{\text {av }}$ is higher than that compared with the case of using the PCS. Since the average fidelity can reach to unit, the second protocol is more suitable than the first one for quantum teleportation of a coherent state using the non-Gaussian entangled resource is the PAASTMPCS.

\section{Acknowledgments}

This research is funded by Vietnam National Foundation for Science and Technology Development (NAFOSTED) under grant number 103.01-2018.361.

\section{Appendix}

\section{A Derivation of Eq. (10)}

Substituting the density operator $\hat{\rho}$ in Eq. (9) to Eq. (8), then expanding a coherent state $|\gamma\rangle_{x}$ in terms of a Fock state as $|\gamma\rangle_{x}=e^{-|z|^{2} / 2} \sum_{k}\left(z^{k} / \sqrt{k !}\right)|k\rangle_{x}$ with $x=\{a, b\}$, we get

$$
\begin{aligned}
W= & \frac{4 e^{2\left(\left|\alpha_{a}\right|^{2}+\left|\alpha_{b}\right|^{2}\right)}}{\pi^{4}} \sum_{m, n=0}^{\infty} \frac{C_{n ; k, h}(\xi) C_{m, k, h}^{*}(\xi)(-1)^{k+h}}{\sqrt{(n+h) !(m+h !(n+k) !(m+k) !}} \\
& \times \int d^{2} \gamma_{a} d^{2} \gamma_{b} e^{2 \gamma_{a}^{*} \alpha_{a}+2 \gamma_{b}^{*} \alpha_{b}-2 \gamma_{a} \alpha_{a}^{*}-2 \gamma_{b} \alpha_{b}^{*}} \\
& \times e^{-\left|\gamma_{a}\right|^{2}-\left|\gamma_{b}\right|^{2}}\left(\gamma_{a}^{*}\right)^{n+k} \gamma_{a}^{m+k}\left(\gamma_{b}^{*}\right)^{n+h} \gamma_{b}^{m+h} .
\end{aligned}
$$

We re-write the Eq. (34) as follows

$$
\begin{aligned}
W= & \frac{4 e^{2\left(\left|\alpha_{a}\right|^{2}+\left|\alpha_{b}\right|^{2}\right)}}{\pi^{2}} \sum_{m, n=0}^{\infty} \frac{C_{n ; k, h}(\xi) C_{m, k, h}^{*}(\xi)(-1)^{k+h}}{\sqrt{(n+h) !(m+h !(n+k) !(m+k) !}} \\
& \times \frac{1}{\pi} \int d^{2} \gamma_{a} e^{-\left|\gamma_{a}\right|^{2}+2 \gamma_{a}^{*} \alpha_{a}-2 \gamma_{a} \alpha_{a}^{*}\left(\gamma_{a}^{*}\right)^{n+k} \gamma_{a}^{m+k}} \\
& \times \frac{1}{\pi} \int d^{2} \gamma_{b} e^{-\left|\gamma_{b}\right|^{2}+2 \gamma_{b}^{*} \alpha_{b}-2 \gamma_{b} \alpha_{b}^{*}}\left(\gamma_{b}^{*}\right)^{n+h} \gamma_{b}^{m+h} \\
= & \frac{8 e^{2\left(\left|\alpha_{a}\right|^{2}+\left|\alpha_{b}\right|^{2}\right)}}{\pi^{3}} \times \sum_{m, n=0}^{\infty} \frac{C_{n ; k, h}(\xi) C_{m, k, h}^{*}(\xi)(-1)^{k+h}}{\sqrt{(n+h) !(m+h !(n+k) !(m+k) !}} J_{1} J_{2},
\end{aligned}
$$

with

$$
J_{1}=\frac{1}{\pi} \int d^{2} \gamma_{a} e^{-\left|\gamma_{a}\right|^{2}+2 \gamma_{a}^{*} \alpha_{a}-2 \gamma_{a} \alpha_{a}^{*}}\left(\gamma_{a}^{*}\right)^{n+k} \gamma_{a}^{m+k},
$$


and

$$
J_{2}=\frac{1}{\pi} \int d^{2} \gamma_{b} e^{-\left|\gamma_{b}\right|^{2}+2 \gamma_{b}^{*} \alpha_{b}-2 \gamma_{b} \alpha_{b}^{*}}\left(\gamma_{b}^{*}\right)^{n+h} \gamma_{b}^{m+h} .
$$

To calculate $J_{1}$ and $J_{2}$ in Eqs. (36) and (37), we consider an integral

$$
J=\frac{1}{\pi} \int d^{2} \beta e^{-|\beta|^{2}+\alpha \beta^{*}}\left(\beta^{*}\right)^{q} e^{-\alpha^{*} \beta} \beta^{l} .
$$

By using a complex integral $\frac{1}{\pi} \int d^{2} \beta e^{-|\beta|^{2}+\alpha \beta^{*}}\left(\beta^{*}\right)^{n} f(\beta)=(\partial / \partial \alpha)^{n} f(\alpha)$, the integral in Eq. (38) is given as

$$
J=(\partial / \partial \alpha)^{q}\left[e^{-\alpha^{*} \alpha} \alpha^{l}\right]
$$

From the definition of the Laguerre function $L_{n}^{i}(z)=\frac{z^{-i} e^{z}}{n !}(d / d z)^{n}\left(e^{-z} z^{n+i}\right)$, and setting $|\alpha|^{2}=y$, we have $\alpha=y / \alpha^{*}$ or $(\partial / \partial \alpha)^{q}=\left(\alpha^{*}\right)^{q}(\partial / \partial y)^{q}$. Thus the integral in Eq. (38) is given in form

$$
J=q !\left(-|\alpha|^{2}\right)^{-q} L_{q}^{-(-l)-q}\left(|\alpha|^{2}\right)(-1)^{q} \alpha^{l}\left(\alpha^{*}\right)^{q} e^{-|\alpha|^{2}} .
$$

Using the correlation between the Laguerre function and the hypergeometric function

$$
{ }_{2} F_{0}(-n, b ; ;-1 / z)=n !(-z)^{-n} L_{n}^{-b-n}(z)
$$

we have

$$
J=(-1)^{q} \alpha^{l}\left(\alpha^{*}\right)^{q} e^{-|\alpha|_{2}^{2}} F_{0}\left(-q,-l ; ;-1 /|\alpha|^{2}\right) .
$$

From Eq. (42), the integrals $J_{1}$ and $J_{2}$ in Eqs. (36) and (37) are given by

$$
J_{1}=(-1)^{n+k}\left(2 \alpha_{a}\right)^{m+k}\left(2 \alpha_{a}^{*}\right)^{n+k} e^{-\left|2 \alpha_{a}\right|_{2}^{2}}{ }_{2} F_{0}\left(-n-k,-m-k ; ;-1 /\left|2 \alpha_{a}\right|^{2}\right),
$$

and

$$
J_{2}=(-1)^{n+h}\left(2 \alpha_{b}\right)^{m+h}\left(2 \alpha_{b}^{*}\right)^{n+h} e^{-\left|2 \alpha_{b}\right|_{2}^{2}}{ }_{2} F_{0}\left(-n-h,-m-h ; ;-1 /\left|2 \alpha_{b}\right|^{2}\right) .
$$

After putting the Eqs. (43) and (44) to Eq. (35), the Wigner function is determined as

$$
\begin{aligned}
W= & \frac{4 e^{-2\left(\left|\alpha_{a}\right|^{2}+\left|\alpha_{b}\right|^{2}\right)}}{\pi^{2}} \sum_{m, n=0}^{\infty} \frac{C_{n ; k, h}(\xi) C_{m, k, h}^{*}(\xi)}{\sqrt{(n+h) !(m+h !(n+k) !(m+k) !}} \\
& \times\left(2 \alpha_{a} 2 \alpha_{b}\right)^{m}\left(2 \alpha_{a}^{*} 2 \alpha_{b}^{*}\right)^{n}\left|2 \alpha_{a}\right|^{2 k}\left|2 \alpha_{b}\right|^{2 h}{ }_{2} F_{0}\left(-n-k,-m-k ; ;-1 /\left|2 \alpha_{a}\right|^{2}\right) \\
& \times{ }_{2} F_{0}\left(-n-h,-m-h ; ;-1 /\left|2 \alpha_{b}\right|^{2}\right) .
\end{aligned}
$$

Note that $\xi=|\xi| e^{i \phi}, \alpha_{x}=\left|\alpha_{x}\right| e^{i \varphi_{x}}$ with $x=\{a, b\}$, the Wigner function in Eq. (45) becomes

$$
\begin{aligned}
W= & \frac{4 e^{-2\left(\left|\alpha_{a}\right|^{2}+\left|\alpha_{b}\right|^{2}\right)}}{\pi^{2}}\left[\sum_{j=0}^{\infty} \frac{|\xi|^{2 j}(j+k) !}{(j !)^{2}(j+h) !}\right]^{-1} \sum_{m, n=0}^{\infty} \frac{|\xi|^{n+m} e^{i(m-n)\left(\varphi_{a}+\varphi_{b}-\phi\right)}}{n ! m !(n+h) !(m+h) !} \\
& \times\left|2 \alpha_{a}\right|^{n+m+2 k}\left|2 \alpha_{b}\right|^{n+m+2 h}{ }_{2} F_{0}\left(-n-k,-m-k ; ;-1 /\left|2 \alpha_{a}\right|^{2}\right) \\
& \times{ }_{2} F_{0}\left(-n-h,-m-h ; ;-1 /\left|2 \alpha_{b}\right|^{2}\right) .
\end{aligned}
$$

Because the imaginary parts of the Wigner function in Eq. (46) are vanished, so the Wigner function is reduced to

$$
\begin{aligned}
W= & \frac{4 e^{-2\left(\left|\alpha_{a}\right|^{2}+\left|\alpha_{b}\right|^{2}\right)}}{\pi^{2}}\left[\sum_{j=0}^{\infty} \frac{|\xi|^{2 j}(j+k) !}{(j !)^{2}(j+h) !}\right]^{-1} \sum_{m, n=0}^{\infty} \frac{|\xi|^{n+m} \cos \left[(m-n)\left(\varphi_{a}+\varphi_{b}-\phi\right)\right]}{n ! m !(n+h) !(m+h) !} \\
& \times\left|2 \alpha_{a}\right|^{n+m+2 k}\left|2 \alpha_{b}\right|^{n+m+2 h}{ }_{2} F_{0}\left(-n-k,-m-k ; ;-1 /\left|2 \alpha_{a}\right|^{2}\right) \\
& \times{ }_{2} F_{0}\left(-n-h,-m-h ; ;-1 /\left|2 \alpha_{b}\right|^{2}\right) .
\end{aligned}
$$

Obviously, the Wigner function in Eq. (47) coincides with the function in Eq. (10). 


\section{References}

1. Glauber, R.J.: Coherent and Incoherent States of the Radiation Field. Phys. Rev. 131, 2766-2788 (1963)

2. Marian, P., Marian, T.A.: Squeezed states with thermal noise. I. Photon-number statistics. Phys. Rev. A 47, 4474-1486 (1993)

3. Agarwal, G.S., Tara, K.: Nonclassical properties of states generated by the excitations on a coherent state. Phys. Rev. A 43, 492-497 (1991)

4. Jones, G.N., Haight, J., Lee, C.T.: Nonclassical effects in the photon-added thermal state. Quantum Semiclass. Opt. 9, 411-418 (1997)

5. Duc, T.M., Noh, J.: Higher-order properties of photon-added coherent states. Opt. Commun. 281, 2842-2848 (2008)

6. Agarwal, G.S.: Generation of Pair Coherent States and Squeezing via the Competition of Four-Wave Mixing and Amplified Spontaneous Emission. Phys. Rev. Lett. 57, 827-830 (1986)

7. Agarwal, G.S.: Nonclassical statistics of fields in pair coherent states. J. Opt. Soc. Am. B 5, 1940 (1988)

8. Caves, C.M., Schumaker, B.L.: New formalism for two-photon quantum optics. I. Quadrature phases and squeezed states. Phys. Rev. A 31, 3068-3092 (1985)

9. Dodonov, V.V.: 'Nonclassical' states in quantum optics: a 'squeezed' review of the first 75 years. J. Opt. B: Quantum Semiclass. Opt. 4, R1-R33 (2002)

10. Biswas, A., Agarwal, G.S.: Nonclassicality and decoherence of photon-subtracted squeezed states. Phys. Rev. A 75, 032104 (2007)

11. Kitagawa, A., Takeoka, M., Sasaki, M., Chefles, A.: Entanglement evaluation of non-Gaussian states generated by photon subtraction from squeezed states. Phys. Rev. A 73, 042310 (2006)

12. Hu, L.Y., Xu, X.X., Wang, Z.S., Xu, X.F.: Photon-subtracted squeezed thermal state: Nonclassicality and decoherence. Phys. Rev. A 82, 043842 (2010)

13. Ban, M.: Photon statistics of conditional output states of lossless beam splitter. J. Mod. Opt. 43, 1281-1303 (1996)

14. Kim, M.S.: Recent developments in photon-level operations on travelling light fields. J. Phys. B: At. Mol. Opt. Phys. 41, 133001 (2008)

15. Duc, T.M., Dat, T.Q.: Enhancing nonclassical and entanglement properties of trio coherent states by photonaddition. Optik 210, 164479 (2020)

16. Zavatta, A., Viciani, S., Bellini, M.: Quantum-to-Classical Transition with Single-Photon-Added Coherent States of Light. Science 306, 660-662 (2004)

17. Parigi, V., Zavatta, A., Kim, M., Bellini, M.: Probing Quantum Commutation Rules by Addition and Subtraction of Single Photons to/from a Light Field. Science 317, 1890 (2007)

18. Barbieri, M., et al.: Non-Gaussianity of quantum states: An experimental test on single-photon-added coherent states. Phys. Rev. A 82, 063833 (2010)

19. Xu, X.X., Yuan, H.C., Hu, L.Y., Fan, H.Y.: Non-Gaussianity of photon-added-then-subtracted squeezed vacuum state. Optik 123, 16-20 (2012)

20. Walschaers, M., Fabre, C., Parigi, V., Treps, N.: Entanglement and Wigner Function Negativity of Multimode Non-Gaussian States. Phys. Rev. Lett. 119, 183601 (2017)

21. Roy, S., Das, T., Sen(De), A.: Computable genuine multimode entanglement measure: Gaussian versus nonGaussian. Phys. Rev. A 102, 012421 (2020)

22. Duc, T.M., Dinh, D.H., Dat, T.Q.: Higher-order nonclassical properties of nonlinear charge pair cat states. J. Phys. B: At. Mol. Opt. Phys. 53, 025402 (2020)

23. Dat, T.Q., Duc, T.M.: Nonclassical Properties of the Superposition of Three-Mode Photon-Added Trio Coherent State. Int. J. Theor. Phys. 59, 3206-3216 (2020)

24. Takeda, S., Benichi, H., Mizuta, T., Lee, N., Yoshikawa, J., Furusawa, A.: Quantum mode filtering of nonGaussian states for teleportation-based quantum information processing. Phys. Rev. A 85, 053824 (2012)

25. Wang, S., Hou, L.L., Chen, X.F., Xu, X.F.: Continuous-variable quantum teleportation with non-Gaussian entangled states generated via multiple-photon subtraction and addition. Phys. Rev. A 91, 063832 (2015)

26. Duc, T.M., Dat, T.Q., Chuong, H.S.: Quantum entanglement and teleportation in superposition of multiplephoton-added two-mode squeezed vacuum state. Int. J. Mod. Phys. B 34, 2050223 (2020)

27. Chowdhury, P., Pramanik, T., Majumdar, A.S., Agarwal, G.S.: Einstein-Podolsky-Rosen steering using quantum correlations in non-Gaussian entangled states. Phys. Rev. A 89, 012104 (2014)

28. Guo, Y., Ye, W., Zhong, H., Liao, Q.: Continuous-variable quantum key distribution with non-Gaussian quantum catalysis. Phys. Rev. A 99, 032327 (2019)

29. Liao, Q., Guo, Y., Huang, D., Huang, P., Zeng, G.: Long-distance continuous-variable quantum key distribution using non-Gaussian state-discrimination detection. New J. Phys. 20, 023015 (2018) 
30. Yang, Y., Li, F.L.: Entanglement properties of non-Gaussian resources generated via photon subtraction and addition and continuous-variable quantum-teleportation improvement. Phys. Rev. A 80, 022315 (2009)

31. Ouyang, Y., Wang, S., Zhang, L.: Quantum optical interferometry via the photonadded two-mode squeezed vacuum states. J. Opt. Soc. Am. B 33, 1373-1381 (2016)

32. Yang, Y.: Enhancing quantum entanglement by combinations of photon additions and photon subtractions. J. Opt. Soc. Am. B 33, 2545-2551 (2016)

33. Lu, D.M.: Quantum Properties of the State via Operation of Superposition of Photon Subtraction Two Times and Photon Addition Two Times on Two Modes Squeezing Vacuum State. Int. J. Theor. Phys. 57, 2767-2774 (2018)

34. Lee, C.T.: Many-photon antibunching in generalized pair coherent states. Phys. Rev. A 41, 1569-1575 (1990)

35. Li-Yun, H., Hong-Yi, F.: Entanglement Involved in Pair Coherent State Studied via Wigner Function Formalism. Commun. Theor. Phys. 52, 1071-1075 (2009)

36. Gerry, C.C., Mimih, J.: Heisenberg-limited interferometry with pair coherent states and parity measurements. Phys. Rev. A 82, 013831 (2010)

37. Dong, Y.L., Zou, X.B., Guo, G.C.: Generation of pair coherent state using weak cross-Kerr media. Phys. Lett. A 372, 5677-5680 (2008)

38. Gerry, C.C., Mimih, J., Birrittella, R.: State-projective scheme for generating pair coherent states in travelingwave optical fields. Phys. Rev. A 84, 023810 (2011)

39. Agarwal, G.S., Biswas, A.: Quantitative measures of entanglement in pair-coherent states. J. Opt. B: Quantum Semiclass. Opt. 7, 350-354 (2005)

40. Gabris, A., Agarwal, G.S.: Quantum teleportation with pair-coherent states. Int. J. Quantum Inf. 5, 17-22 (2007)

41. Wang, L., Zhao, S.: Round-robin differential-phase-shift quantum key distribution with heralded pair-coherent sources. Quantum Inf. Process 16, 1-15 (2017)

42. Salimi, S., Mohammadzade, A.: D-Concurrence Bounds for Pair Coherent States, Int. J. Theor. Phys. 50, 1444-1450 (2011)

43. Dat, T.Q., Duc, T.M., Chuong, H.S.: J. Phys.: Improvement quantum teleportation via the pair coherent states. Conf. Series 1034, 012004 (2018)

44. Hong, L., Guang-can, G.: Nonclassical properties of photon-added pair coherent states. Acta Phys. Sin. 8, 577-582 (1999)

45. Yuan, H.C., Xu, X.X., Fan, H.Y.: Statistical Properties of the Generalized Photon-Added Pair Coherent State. Int. J. Theor. Phys. 48, 3596-3606 (2009)

46. Chunqing, H. Hong, L.: Statistical properties of photon-added and photon-subtracted pair coherent state. Acta Photon. Sin. 29, 481-486 (2000)

47. Kenfack, A., Zyczkowski, K.: Negativity of the Wigner function as an indicator of non-classicality. J. Opt. B: Quantum Semiclass. Opt. 6, 396-404 (2004)

48. Hoai, N.T.X., Duc, T.M.: Nonclassical properties and teleportation in the two-mode photon-added displaced squeezed states. Int. J. Mod. Phys. B 30, 1650032 (2016)

49. Peres, A.: Separability Criterion for Density Matrices. Phys. Rev. Lett. 77, 1413-1415 (1996)

50. Duan, L.M., Giedke, G., Cirac, J.I., Zoller, P.: Inseparability Criterion for Continuous Variable Systems. Phys. Rev. Lett. 84, 2722-2725 (2000)

51. Hillery, M., Zubairy, M.S.: Entanglement Conditions for Two-Mode States. Phys. Rev. Lett. 96, 050503 (2006)

52. Wang, X.G.: Bipartite entangled non-orthogonal states. J. Phys. A: Math. Gen. 35, 165-173 (2002)

53. Wu, J., Liu, S., Hu, L., Huang, J., Duan, Z., Ji, Y.: Improving entanglement of even entangled coherent states by a coherent superposition of photon subtraction and addition. J. Opt. Soc. Am. B 32, 2299-2307 (2015)

54. Hofmann, H.F., Ide, T., Kobayashi, T.: Fidelity and information in the quantum teleportation of continuous variables. Phys. Rev. A 62, 062304 (2000)

55. Cochrane, P.T., Milburn, G.J., Munro, W.J.: Teleportation using coupled oscillator states. Phys. Rev. A 62, 062307 (2000)

56. Kurucz, Z., Adam, P., Kis, Z., Janszky, J.: Continuous variable remote state preparation. Phys. Rev. A 72, $052315(2005)$ 